\title{
Physiological Implications of Two versus Three Sets in the Development of Quadriceps Muscle Strength in Untrained Men
}

\section{Baker JS ${ }^{1 *}$, Buchan DS ${ }^{1}$, Wong DP², Davies B ${ }^{3}$, Cooper SM${ }^{4}$, Davies M3 and Kilgore $\mathrm{L}^{1}$}

${ }^{1}$ Institute for Clinical Exercise \& Health Science, University of the West of Scotland, Hamilton, ML3 OJB, Scotland, UK ${ }^{2}$ Human Performance Laboratory, Technological and Higher Education Institute of Hong Kong, Hong Kong, P.R. China

${ }^{3}$ Health and Exercise Science Research Unit, School of Applied Sciences, University of South Wales, Pontypridd, CF37 1DL, Wales, UK

${ }^{4}$ Cardiff School of Sport, Cardiff Metropolitan University, Cardiff CF23 6XD, Wales, UK

\section{Abstract}

Background: The purpose of this study was to determine if differences in strength gain are apparent following resistance training at two different exercise volumes (2-sets versus 3-sets).

Methods: Seven men (age=21.6 \pm 1.5 ) completed the study. Each subject trained one leg in the leg extension exercise using two sets and trained the other using three sets. Subjects were randomly assigned to two groups then treatments were randomly assigned to groups to minimize bias: one group assigned two sets right leg and three sets left leg ( $3 L 2 R ; n=3)$, one group assigned three sets right leg and two sets left leg (3R $2 L ; n=4)$. One repetition maximum (1RM) was determined for each leg of each participant for the leg extension prior to the three sessions per week and six week duration training program. Each set included in training consisted of six repetitions at a workload of $80 \% 1 \mathrm{RM}$ during each session. 1RM was tested after 2 and 4 weeks allowing for training workloads to be adjusted to $80 \%$ of current 1RM to apply the principle of progression.

Results: Significant differences were apparent when comparing pre- and post-training absolute 1RM measures for both the two set and three set legs $(p<0.05)$. There was a significant difference in magnitude of change between the two set and three set legs, $12.6 \mathrm{~kg}$ versus $19.4 \mathrm{~kg}$ respectively $(p=0.02)$.

Conclusion: While six weeks of either two or three sets of the leg extension exercise training both significantly increase quadriceps strength, the three set configuration creates a significantly larger magnitude of change compared to two sets.

Keywords: Strength training; Muscle adaptation; 1RM

\section{Introduction}

Resistance training has been well established as a highly effective method for improving muscular strength for more than half a century [1-4]. Resistance training is beneficial to the development of musculoskeletal fitness and is known to decrease the risk of injury and disease to the individual, both cardiovascular and orthopaedic in the elderly [5-7]. There is further evidence that low muscle strength significantly compromises mobility and function in the elderly increases the prevalence of obesity and is associated with increased allcause mortality in both the elderly and young [8-11].

The manner in which strength training programs should be structured to increase strength and reap functional benefits continues to be a source of practitioner and scientific debate. Resistance training and its effects are dependent on several factors, including the intensity and frequency of training, rest interval duration and exercise volume. In relation to volume of training, although the number of sets and repetitions prescribed for strength gain is not precisely known $[3,12,13]$. The prevailing consensus, derived from studies generally examining programs using 1-set or 3-sets, is that execution of multiple sets of an exercise is required to elicit optimal increases in muscular strength [2,14-16]. Typically this is the general information included in coaching and fitness professional instructional materials. The validity of this onesize-fits-all prescription can be questioned as several studies are present in the literature suggesting that single set programs may be as efficient as multiple sets in the development of muscular strength [13,17-22].

The concept that single set training is as strong of a stimulus for strength gain as multiple sets was presented in the paper by Carpinelli and Otto [2]. They suggested, based on the literature they reviewed, if three sets does not succeed in bringing about significantly greater increases in strength than a single set then it seems plausible that considerable time and effort could be spared by weight trainees by simply performing a single set per exercise per training session. It must be noted that numerous professionals and scientists claimed the review was biased in papers included [23]. Further, a meta-analysis by Krieger suggests that multiple sets produce $40 \%$ greater hypertrophy than single sets in both untrained and trained subjects [24]. Although hypertrophy is a different training goal than strength gain, the findings suggest that there is an authentic difference in anatomical and physiological adaptations between single and multiple set programs.

A number of training studies report similarity in results after single and multiple set programs undertaken for the short term (7-10 weeks in duration) [17,19-21]. These findings have come under criticism for being too short in duration to elicit statistically significant changes. The short term nature of these studies seemingly provides some of an explanation as to why single sets, in many authors' work, indicate similar strength improvements with one or multiple sets. Galvao and Taaffe agree with this, stating that many programs do not exceed a 14 week time course, which may limit the potential for strength gain [12]. To address this concern De Hoyos et al. and Vincent et al. conducted studies that took place over a period of 25 weeks, and produced similar conclusions to the shorter tem studies - that both single and

*Corresponding author: Julien Baker, Institute for Clinical Exercise \& Health Science, University of the West of Scotland, Hamilton, ML3 0JB, Scotland, UK, Tel: 01698 283100; Fax: 01698 894404; E-mail: julien.baker@uws.ac.uk

Received September 04, 2013; Accepted September 28, 2013; Published December 05, 2013

Citation: Baker JS, Buchan DS, Wong DP, Davies B3, Cooper SM, et al. (2013) Physiological Implications of Two versus Three Sets in the Development of Quadriceps Muscle Strength in Untrained Men. J Sports Med Doping Stud 3: 132. doi:10.4172/2161-0673.1000132

Copyright: @ 2013 Baker JS, et al. This is an open-access article distributed under the terms of the Creative Commons Attribution License, which permits unrestricted use, distribution, and reproduction in any medium, provided the original author and source are credited. 
multiple set groups improved strength to a significant degree, but that any differences noted between the groups were not large enough to be statistically significant $[18,22]$.

It has been suggested by Fleck and Kraemer that resistance training consisting of single sets may well be suitable for beginning trainees commencing a strength training program and that multiple sets will be most advantageous in terms of producing physiological adaptations once an initial fitness level has been reached through the initial single set regime [3]. This concept was refined and linked to Selye's general adaptation syndrome by Rippetoe and Kilgore through a definition of training progression (novice, intermediate, advanced and elite) who respond to general training parameters at different rates and with differing magnitudes of strength gain $[25,26]$. Essentially, exposure to strength training of previously untrained subjects will produce similar rates and magnitudes to strength gain across all resistance training procedures. Beginning trainees respond to all programs as a general stimulus for adaptation thus the similarity of response. More advanced trainees require more specific sources of stress (i.e., multiple sets) to produce fitness gains. A paper by Silvester et al. analysed single set versus multiple set programs and are in agreement with this concept, finding that single set protocols result in similar strength increases as multiple sets over short durations of training in untrained subjects, but that as the individual's strength was enhanced multiple set resistance training protocols were more advisable and effective [19]. This finding is suggestive that beyond the short term nature of studies proposing that single set training is as effective as those using multiple sets another concern is the training progression status of the subjects. It is often the case that untrained subjects are the focal point of the investigations thus providing another factor needing attention and description in experimentation. Studies using novice trainees as subjects are quite valid and informative, however their findings must be restricted to similar populations, those with no or limited training experience.

There has been a large degree in dissimilarity in the repetition ranges and set numbers used in the one versus multiple set investigations to date. Kraemer et al. [27] illustrated that significantly greater increases in 1RM squat ability were acquired by subjects undertaking a multiple set program $(3 \times 10$ repetitions $)$ in comparison to those performing a single set protocol ( $1 \times 12$ repetitions), where both groups trained three times a week for a 14 week period. Schlumberger et al. [28] also presented evidence that superior strength gains were associated with those that trained with 3 sets in comparison to those using a single set resistance training program, with a study focused on leg extension strength improvements. Conflicting evidence to this was provided by Ostrowski et al. [29], whose study on recreational weightlifters it was concluded after a 10 week program that three sets per exercise was as effective as 6 or 12 sets for inducing strength gains. Hass et al. [13] supplied further support for single set training being sufficient for strength increases in recreational weightlifters, with a study involving two groups, one of which performed 1 set of 8-12 repetitions and the other 3 sets of $8-12$ repetitions both at $70 \%$ of $1 \mathrm{RM}$. The results obtained indicated that leg extension strength increased significantly in both groups from pre-training to midpoint measures and from midpoint to post-training measures, but also that at no time during the program did the differences in leg extension 1RM between the groups differ significantly. Conclusions regarding multi-set versus single set training that are based on differing volumes (repetitions and sets) and intensities is problematic as they have been shown to be linked to differential physiological adaptations in muscle [30].

Due to the variance in approaches to constructing experimental training programs, there has been contrasting evidence to date concerning single versus multiple set resistance training. The strength

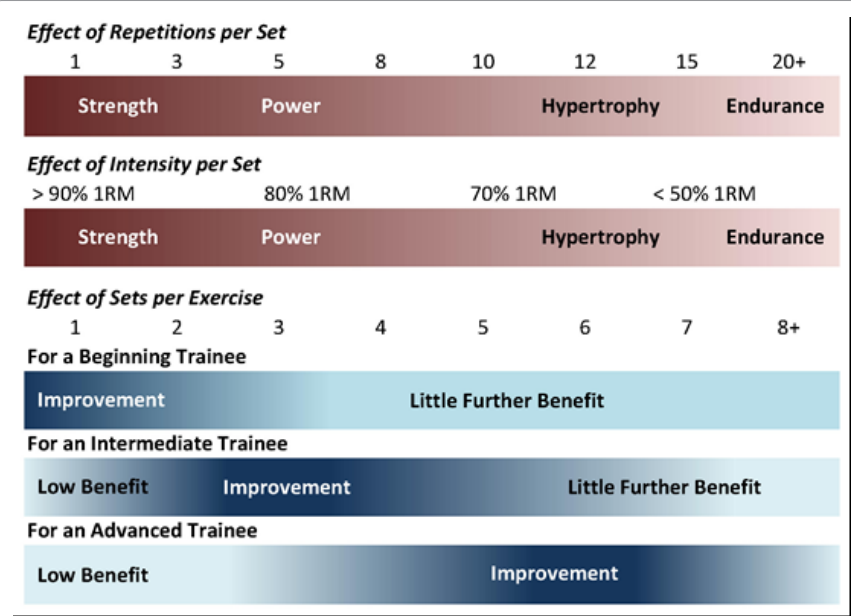

Figure 1: Box-whisker-plot of the ratio testosterone $(T)$ to epitestosterone $(E)$ at baseline (V2) and end of trial after 12 weeks (V4) for both treatment groups. Shown are the median (small line within the box) with interquartile range ICR (upper and lower quartile), $5^{\text {th }}$ and $95^{\text {th }}$ percentiles and minimum and maximum values $(+)$.

continuum that is an operational construct in professional strength training practice has been supported in the literature (Figure 1) [30]. However a complete and systematic exploration of repetition and set schemes need to be undertaken to more fully establish its accuracy. The most complete analysis of the effects of different set numbers was that of Berger [1]. Given the limited literature in existence regarding discriminating between the differences in the amount of strength two and three set program organizations induce, this set range was compared, as it has received the least attention but may have a profound utility in lower volume training programmes. The present small-scale study proposed to examine strength changes in the quadriceps after four weeks of training in beginning weight trainees. A within subjects method was used (right and left legs receiving differing training) to control for inter-individual variance.

The most common repetition per set recommendations for beginners currently in use are for five repetitions and 6-12 repetitions [26,31,32]. The present study examined a 6 repetition per set organization, similar to popular and exercise authority recommendations. The experimental hypothesis was that three sets of exercise would produce a larger change in strength than two sets of exercise (Figure 1).

\section{Materials and Methods}

\section{Subjects}

Ten male subjects volunteered for this study, ranging from 19-24 years of age. All 10 subjects were apparently healthy and were beginners and not involved in any other type of training for strength development, in order to ensure that no muscle strength gains were due to any means other than those set out in this investigation. In addition to this, no other leg exercises or leg training (other than normal ambulation) was performed by the participants either within the study or outside of it throughout the entire program duration. The descriptive data of the subjects are presented in Table 1. Prior to the commencement of the study the participants were fully instructed of what was required of them and they each gave written informed consent, with the knowledge that at any time they could voluntarily withdraw from the investigation.

\section{Experimental design}

This investigation centred on the increases in quadriceps strength 
Citation: Baker JS, Buchan DS, Wong DP, Davies B3, Cooper SM, et al. (2013) Physiological Implications of Two versus Three Sets in the Development of Quadriceps Muscle Strength in Untrained Men. J Sports Med Doping Stud 3: 132. doi:10.4172/2161-0673.1000132

Page 3 of 5

\begin{tabular}{|c|c|c|c|}
\hline Group & Age (y) & Height (cm) \\
\hline $3 R \quad 2 L(n=4)$ & $21.8(2.1)$ & $177.1(3.7)$ \\
\hline $3 L 2 R(n=3)$ & $21.3(0.6)$ & $181.6(6.5)$ \\
\hline
\end{tabular}

Table 1: Subject descriptive data presented as means (standard deviation).

\begin{tabular}{|c|c|c|c|c|c|c|}
\hline 1RM strength & Pre-Training & 2-week & 4-week & 6-week & \% change & Effect size between pre- and post-test \\
\hline 2-set leg & $52.0(11.1)$ & $57.1(10.3)$ & $61.1(10.8)$ & $64.6(9.1)^{\star}$ & 24.2 \\
\hline 3-set leg & $51.4(12.9)$ & $58.9(13.0)$ & $65.7(14.8)$ & $70.9(15.6)^{\star}$ & 37.9 & 1.24 \\
\hline
\end{tabular}

'Denotes significant differences of pre- to post-training $1 \mathrm{RM}$ values within legs $(\mathrm{P}<0.05)$. Table 2: Changes in mean $1 \mathrm{RM}$ over the experimental period. All results in kg $(\mathrm{SD})$.

\begin{tabular}{|c|c|c|}
\hline Subject & 2-set leg & 3-set leg \\
\hline 1 & 20 & 40 \\
\hline 2 & 12 & 16 \\
\hline 3 & 16 & 24 \\
\hline 4 & 8 & 20 \\
\hline 5 & 8 & 8 \\
\hline 6 & 16 & 16 \\
\hline 7 & 8 & 12 \\
\hline Mean (SD) & $12.6(4.9)$ & $19.4(10.4)^{\star}$ \\
\hline
\end{tabular}

Table 3: Comparison of magnitude of strength gain in legs trained with 2 sets and 3 sets of the leg extension exercise using $80 \% 1 \mathrm{RM}$ for six weeks. Results in kg (SD). ${ }^{*}$ indicates statistically different value from 2 -set training protocol $(p<0.05)$

obtained through different protocols of resistance training. As part of the experiment, each subject was required to train both their right and left leg quadriceps muscles, but in a dissimilar fashion. One leg was trained with two sets of six repetitions in the leg extension exercise and the other with three sets of six repetitions per training session. The 10 subjects were randomly assigned into two groups: (1) five subjects trained using three sets with the right leg and two sets with the left (3R 2L) and (2) five subjects trained using two sets with the right leg and three sets with the left leg $(3 \mathrm{~L} 2 \mathrm{R})$. This orientation was selected to reduce bias in the test regarding right or left leg dominance in subjects. The program lasted for a period of six weeks with three sessions being performed each week (Monday-Wednesday-Friday at the same time of day), with at least 48 hours between sessions. The six weeks of training were consecutive to ensure that no reversible effects to training could occur during the program and that the subjects were training at an identical frequency. The training sessions were undertaken on a standard leg extension exercise machine (Power sports International Ltd., UK) adjusted according to tibial length so that the machine pad rested at a level just above the level of the lateral malleolus of each subject. Subjects sat on the machine seat in a position where their gastrocnemius was approximately $1-2 \mathrm{~cm}$ in advance of the forward edge of the seat. Each repetition began with the tibia perpendicular to the floor, went through complete extension (180 degrees) of the knee, and ended with return of the tibia to a perpendicular position.

One repetition maximum (1RM) for leg extension of each leg of each subject was tested prior to the commencement of training as a baseline for the later comparison of strength gains. Two further 1RM tests were conducted as strength re-tests after the second and fourth weeks of training and again at the end of the program (sixth week).

During each training session both legs were separately exercised at a workload of $80 \%$ of each subject's 1RM, as calculated from their preprogram 1RM measurements for each respective leg. Following re-test measurements after two and four weeks the workload of the training sessions was increased to $80 \%$ of the new 1 RM result to account for any increases in strength occurring in that time period. Each set consisted of six repetitions with a standardized two minute rest interval observed between sets. Before any training session, a standard warm-up was undertaken by all participants consisting of 10-12 repetitions on the leg extension machine at a low weight appropriate to each individual, or approximately $50 \% 1 \mathrm{RM}$.

\section{Statistical analysis}

As the research hypothesis assumed directionality in strength gain, as a consequence critical values were established using one-tailed tests (i.e., strength gain). Two sets of data (pre-experimental 1RM versus post-experimental 1RM; magnitude of 1RM change) were analysed using paired t-tests to determine if any significant differences existed between the two. Level of significance was set $a$ priori at $\mathrm{p}<0.05$. Mean improvements in strength for both the two set and three set legs were calculated from initial and end 1RM values.

\section{Results}

Of the ten original subjects recruited for this study, only seven successfully completed the entire six week training period. Two subjects voluntarily withdrew from the study for personal reasons and one withdrew due to an injury sustained outside of the investigation. As only complete data sets could be used, data from seven subjects was included in the final analysis. Of the remaining seven participants, three were part of the $3 \mathrm{~L} 2 \mathrm{R}$ group, and the other four were in the $3 \mathrm{R} 2 \mathrm{~L}$ group.

Table 2 illustrates the mean and standard deviations (SD) 1RM for two sets versus three sets of unilateral leg extension training for the seven subjects. The results are presented in $1 \mathrm{RM}$ means (SD) for pretraining, following two weeks, four weeks, and at the end of the six week training program. Intermediate $1 \mathrm{RM}$ values (second and fourth weeks) were only used for program modification and not included in statistical analysis of the efficacy of the two or three set training organizations.

Both the two set and three set trained legs experienced large increases in $1 \mathrm{RM}$ of $24.2 \%$ and $37.9 \%$ respectively. Both findings are statistically significant when comparing pre-training and post-training data $(\mathrm{p}<0.05)$. Comparison of these pre-training and post-training $1 \mathrm{RM}$ values between resulted in a $\mathrm{p}=0.14$, a value that was not statistically significant.

However, as the intent of the project was to detect any differences between 2 or 3 set groups in the magnitude of change in 1RM following training (Table 3 ). The magnitude of change elicited was calculated, 
five $(71.4 \%)$ of the seven subjects increased their 1RM differentially between legs, favouring the three set intervention. Two subjects (28.6\%) experienced similar results between exercise treatments. The average difference in strength gain between two set trained and three set trained legs was $6.9 \mathrm{~kg}( \pm 7.2)$ in favour of three set trained legs. This differential in magnitude of $1 \mathrm{RM}$ gain was statistically significant ( $\mathrm{p}=0.02$ ) suggesting that there is an authentic difference in the effect of increased training volume ( 2 sets versus 3 sets) on strength gain.

\section{Discussion}

When comparing pre- and post-training strength increases following the 6 week resistance training program, it can be statistically shown that both training with 2 sets and 3 sets per session elicit significant strength gains in the quadriceps muscles of untrained men $(\mathrm{p}<0.05)$. This finding was expected when considering work of other authors having presented similar conclusions [2,13,18,22]. There have been mixed findings in the literature supporting higher volumes (3 set) being superior to lower volumes (1 set) of training [1,14,33]. Alternatively there is data suggesting that single set training is just as efficient in eliciting strength gain as multiple sets of exercise $[13,20]$. Most available studies have focused upon comparison of single set exercise organizations and three or more sets of exercise. This small scale study attempted to explore the controversial proposition that that exercise volume (number of sets completed) is directly related to magnitude of strength gain and to examine an intermediary number of sets of exercise, two versus three sets, thus adding further information on the validity of the frequently proposed strength continuum.

The variability in previous findings may have been induced by lack of attention to protocol design. Methods for ensuring equity and accuracy included adjusting the exercise machine relative to specific anatomical landmarks on the individual, employing identical exercise postures between subjects, and ensuring that the range of motion was identical between subjects. Some studies have come under criticism as their results may have been affected by confounding variables such as inappropriate or irregular rest intervals. In this study rest intervals between sets were restricted to two minutes [13,14,20,27,33].

The premise of modern strength training programs is largely based on the general adaptation syndrome theory forwarded by Selye [25]. In Selye's original synthesis, exhaustive exercise was considered a noxious stress, thus the theory's application in modern exercise prescription has to be carefully considered and modified. The crux of the general adaptation syndrome, in application to exercise, is that exercise must induce a non-lethal disruption of homeostasis in a target cell, tissue, organ or system and then the organism must be allowed sufficient opportunity to recover and adapt. This adaption enhances survivability and carries with it improved fitness. The magnitude of loading (sets of exercise at $80 \% 1 \mathrm{RM}$ here) is the external stress that requires adaptation. The very similar nature of the load used in this project, one set versus two sets, and the resulting findings indicate that the body is very capable of an exquisitely sensitive detection of loading magnitudes and adaptive variation.

While the present study found significant differences in elicited strength gain between two and three sets, the examination of differences between immediately sequential ordinal numbers of sets is not common in the literature. Few papers have tested for differences in strength gain between one and two sets and two and three sets. The primary rationale for this exclusion is that in Berger's seminal work comparing one, two, and three sets of exercise it was concluded that one and two sets of exercise is not as effective as three sets [1]. So it is common that single sets are compared to larger multiples of sets $[34,35]$. Our study is similar to that of Berger in that our findings were that three sets are superior to two sets. In the paper by Limke et al. a discernibly different strength adaption was not seen in trainees following single or two set training protocols [36]. Combined, the present data and that of Limke support the findings presented by Berger. It could be suggested, based on these observations, that a single set and two sets of weighted exercise does not disrupt muscle homeostasis as effectively as three sets as evidenced by the larger magnitude of 1RM increase after three-set training.

There is a limitation in this study that must be considered, the attrition of subjects resulted in a very low final $n$. Despite the low subject numbers, the results are paired and genetically matched. The right and left legs of the subjects each received different training interventions and reduced intra-subject variability between treatments. This is the same genetic pairing approach that enabled elucidation of the mechanism of hypertrophy by Gollnick et al. in 1981 [37]. This pairing enables a more robust comparison of data and strengthens the ability to make relevant conclusions regarding the two treatments.

The magnitude of increase in 1RM was significantly larger for the three set intervention compared to two sets (mean of $19.4 \mathrm{~kg}$ compared to $12.6 \mathrm{~kg}$ ). These increases may be specific to machine exercise in magnitude and time course. Free weights represent a potentially larger homeostatic disruption than possible with machine weights, as machines have pre-determined movement patterns not requiring contributions from synergists active in coordinated movement of free weights or in the maintenance of balance. The use of machine weights may also have affected both 1RM testing and training progression. The leg extension machine used was designed to allow resistance increases in $4 \mathrm{~kg}$ increments. This placed a limitation in sensitivity of 1RM testing as any strength gain of less than $4 \mathrm{~kg}$ would have been undetectable. This could have resulted in an under-representation of 1RM. The limitation in increment to $4 \mathrm{~kg}$ may also have affected loading and resulted in lower weights being used in training than potentially supported by subject adaptive capacity. Neither of these issues could have been avoided given the equipment available. Future studies should consider using free weight exercises as weight addition increments can be accommodated down to $0.25 \mathrm{~kg}$.

\section{Conclusions and Practical Implications}

This study demonstrated that a three times per week and six week duration machine resistance training program, using six repetitions and either two sets or three sets of leg extension exercise with $80 \% 1 \mathrm{RM}$ will elicit significant increases in quadriceps muscle strength. However, three sets of exercise increased the magnitude of strength gain by an additional $53.9 \%$ over two sets of exercise. These findings suggest that practitioners can indeed produce significant strength gains using two sets, which may be of value to trainers and coaches working with time limited individuals. However, three sets were clearly more effective in producing a change in strength. Given that the additional time to do a third set is two additional minutes for recovery and less than a minute for conduct of the third exercise set, this may mitigate against the rationale of not doing a third set in the interest of saving time.

\section{References}

1. Berger RA (1962) Effect of varied weight training programs on strength Research Quarterly 33: 168-181.

2. Carpinelli RN, Otto RM (1998) Strength training. Single versus multiple sets Sports Med 26: 73-84.

3. Fleck S, Kraemer W (1997) Designing resistance-training programs. ( $2^{\text {nd }}$ edn) Human Kinetics, Champaign, USA. 
Citation: Baker JS, Buchan DS, Wong DP, Davies B3, Cooper SM, et al. (2013) Physiological Implications of Two versus Three Sets in the Development of Quadriceps Muscle Strength in Untrained Men. J Sports Med Doping Stud 3: 132. doi:10.4172/2161-0673.1000132

4. McDonagh MJ, Davies CT (1984) Adaptive response of mammalian skeletal muscle to exercise with high loads. Eur J Appl Physiol Occup Physiol 52: 139155

5. Pollock ML, Wilmore JH (1990) Exercise in health and disease. (2nd Edn), W.B. Saunders, Philadelphia, USA

6. Artero EG, Lee DC, Lavie CJ, España-Romero V, Sui X, et al. (2012) Effects of muscular strength on cardiovascular risk factors and prognosis. J Cardiopulm Rehabil Prev 32: 351-358.

7. Fiatarone MA, O'Neill EF, Ryan ND, Clements KM, Solares GR, et al. (1994) Exercise training and nutritional supplementation for physical frailty in very elderly people. N Engl J Med 330: 1769-1775.

8. Marsh AP, Rejeski WJ, Espeland MA, Miller ME, Church TS, et al. (2011) Muscle strength and BMI as predictors of major mobility disability in the Lifestyle Interventions and Independence for Elders pilot (LIFE-P). J Gerontol A Biol Sci Med Sci 66: 1376-1383.

9. Jackson AW, Lee DC, Sui X, Morrow JR Jr, Church TS, et al. (2010) Muscular strength is inversely related to prevalence and incidence of obesity in adult men. Obesity (Silver Spring) 18: 1988-1995.

10. Ruiz JR, Sui X, Lobelo F, Morrow JR Jr, Jackson AW, et al. (2008) Association between muscular strength and mortality in men: prospective cohort study. BMJ 337: a439.

11. Ortega FB, Silventoinen K, Tynelius P, Rasmussen F (2012) Muscular strength in male adolescents and premature death: cohort study of one million participants. BMJ 345: e7279.

12. Galvão DA, Taaffe DR (2004) Single- vs. multiple-set resistance training: recent developments in the controversy. J Strength Cond Res 18: 660-667.

13. Hass CJ, Garzarella L, de Hoyos D, Pollock ML (2000) Single versus multiple sets in long-term recreational weightlifters. Med Sci Sports Exerc 32: 235-242.

14. Kraemer WJ (1997) A series of studies: The physiological basis for strength training in American football: Facto over philosophy. J Strength Cond Res. 11 $131-142$

15. Kraemer WJ, Ratamess N, Fry AC, Triplett-McBride T, Koziris LP, et al. (2000) Influence of resistance training volume and periodization on physiological and performance adaptations in collegiate women tennis players. Am J Sports Med 28: $626-633$

16. Marx JO, Ratamess NA, Nindl BC, Gotshalk LA, Volek JS, et al. (2001) Lowvolume circuit versus high-volume periodized resistance training in women. Med Sci Sports Exerc 33: 635-643.

17. De Hoyos DV, Herring D, Garzarella L, Weber G, et al. (1997) Effect of strength training volume on the development of strength and power in adolescent tennis players. Med Sci Sports Exerc 29: 164.

18. De Hoyos DV, Herring D, Abe T, Garzarella L (1998) Effect of 6 months of highor low-volume resistance training on muscular strength and endurance. Med Sci Sports Exerc 30: 165

19. Silvester LJ, Stiggins C, McGown C, Bryce R (1982) Effect of variable resistance and free-weight training programs on strength and vertical jump. Natl Str Cond Assoc J 3: 30-33.

20. Stadler Jr VL, Stubbs NB, Vokovich M D (1997) A comparison of a 2-day and 3-day per week resistance training program on strength gain in older adults. Med Sci Sports Exerc. 29: 254

21. Tim Stowers, Jim McMillan, Dwight Scala, Voris Davis, Dennis Wilson, et al (1983) The short term effects of three different strength-power training modes. Natl Str Cond Assoc J 5: 24-27.

22. Vincent K, DeHoyos D, Garzarella L, Hass C, Nordman M, et al. (1998) Relationship between indices of knee extension strength before and after resistance training. Med Sci Sports Exerc 30: 163.
23. Byrd R, Chandler TJ, Conley MS, Fry AC, Haff GG, et al. (1999) Strength training: single versus multiple sets. Sports Med 27: 409-416.

24. Krieger JW (2010) Single vs. multiple sets of resistance exercise for muscle hypertrophy: a meta-analysis. J Strength Cond Res 24: 1150-1159.

25. Selye H (1936) A syndrome produced by diverse nocuous agents. Nature 138 32.

26. Rippetoe M, Kilgore $L$ (2009) Practical Programming for Strength Training ( $2^{\text {nd }}$ Edn), Aasgaard Company, Wichita Falls TX, USA.

27. Kramer JB, Stone MH, O'Bryant HS, Conley MS, Robert L Johnson, et al (1997) Effects of single vs. multiple-sets of weight training: impact of volume, Intensity, and variation. J Strength Cond Res. 11: 143-147.

28. Schlumberger A, Stec J, Schmidtbleicher D (2001) Single- vs. multiple-set strength training in women. J Strength Cond Res 15: 284-289.

29. Ostrowski K, Wilson G, Weatherby R, Murphy P, Andrew D Lyttle (1997) The effects of weight training volume on hormonal output and muscular size and function. J Strength Cond Res 11: 154.

30. Campos GE, Luecke TJ, Wendeln HK, Toma K, Hagerman FC, et al. (2002) Muscular adaptations in response to three different resistance-training regimens: specificity of repetition maximum training zones. Eur J Appl Physio 88: $50-60$

31. Starr B (2007) Strongest Shall Survive: Strength training for football. Five Starr Productions, Baltimore MD, USA

32. American College of Sports Medicine (2009) American College of Sports Medicine position stand. Progression models in resistance training for healthy adults. Med Sci Sports Exerc 41: 687-708.

33. Rhea MR, Alvar BA, Ball SD, Burkett LN (2002) Three sets of weight training superior to 1 set with equal intensity for eliciting strength. J Strength Cond Res 16: $525-529$

34. Kelly SB, Brown LE, Coburn JW, Zinder SM, Gardner LM, et al. (2007) The effect of single versus multiple sets on strength. J Strength Cond Res 21: 10031006.

35. Munn J, Herbert RD, Hancock MJ, Gandevia SC (2005) Resistance training for strength: effect of number of sets and contraction speed. Med Sci Sports Exerc 37: 1622-1626.

36. Limke JC, Rainville J, Peña E, Childs L (2008) Randomized trial comparing the effects of one set vs two sets of resistance exercises for outpatients with chronic low back pain and leg pain. Eur J Phys Rehabil Med 44: 399-405.

37. Gollnick PD, Timson BF, Moore RL, Riedy M (1981) Muscular enlargement and number of fibers in skeletal muscles of rats. J Appl Physiol Respir Environ Exerc Physiol 50: 936-943. 